Meta

Journal des tradlucteurs

Translators' Journal

\title{
Quelques termes sur les moteurs thermiques
}

\section{Jacques Lethuillier}

Volume 33, numéro 3, septembre 1988

URI : https://id.erudit.org/iderudit/003496ar

DOI : https://doi.org/10.7202/003496ar

Aller au sommaire du numéro

Éditeur(s)

Les Presses de l'Université de Montréal

ISSN

0026-0452 (imprimé)

1492-1421 (numérique)

Découvrir la revue

Citer cet article

Lethuillier, J. (1988). Quelques termes sur les moteurs thermiques. Meta, 33(3),

398-407. https://doi.org/10.7202/003496ar d'utilisation que vous pouvez consulter en ligne.

https://apropos.erudit.org/fr/usagers/politique-dutilisation/ 


\section{ÉTUDES TERMINOLOGIQUES ET LINGUISTIQUES}

\section{QUELQUES TERMES SUR LES MOTEURS THERMIQUES}

\section{adiabatique}

Se dit d'une transformation subie par un système thermodynamique qui n'est pas accompagnée d'un échange de chaleur avec le milieu extérieur.

\section{admission}

Entrée du fluide moteur dans l'organe de détente d'une machine thermique motrice, qui peut se faire selon un processus continu (turbine à vapeur) ou périodique (moteur à combustion interne, machine à vapeur alternative).

\section{allumage - firing, ignition}

Amorçage d'une réaction de combustion, qui correspond notamment à une fonction essentielle dans les moteurs à combustion intermittente du fait qu'elle présente la partìcularité d'être périodique.

\section{apport de chaleur - heat input}

Au cours du cycle thermodynamique caractérisant le fonctionnement d'un moteur thermique, phase pendant laquelle est fournie au fluide moteur de la chaleur qui sera partiellement transformée en travail pendant la détente. L'apport de chaleur contribue à recréer ou à entretenir les conditions propices à la détente. $\mathrm{Il}$ précède donc la détente et peut éventuellement se poursuivre pendant la détente, qu'il a alors pour effet de freiner. Les modalités d'apport de chaleur sont variables : par combustion continue selon un processus de transfert direct (turbine à gaz) ou indirect avec échangeur de chaleur (turbine à vapeur), par combustion intermittente (moteur à combustion interne), ou sans combustion (moteur solaire).

\section{autoinflammation - self-ignition}

1. Défaut de fonctionnement observé dans les moteurs à allumage commandé, se traduisant par un allumage intempestif ou incontrôlé du mélange explosif en cours de compression. Diverses causes peuvent être à l'origine de ce dérèglement (points chauds, point d'inflammation trop bas du carburant, etc.).

2. Dans un moteur diesel, inflammation spontanée du combustible se produisant au moment où il est incorporé à la charge d'air préalablement portée à très haute température au cours de la phase de compression.

charge - charge

Quantité de mélange carburé ou d'air pur introduite dans la chambre de combustion d'un moteur à explosion ou d'un moteur diesel pendant l'admission (aspiration provoquée par la descente du piston à l'intérieur du cylindre). 
circuit d'injection - injection system

Sur un moteur diesel, ensemble d'éléments destinés à introduire à tour de rôle dans les différents cylindres, au moment opportun au cours de chaque cycle, une quantité de combustible appropriée au régime de fonctionnement, sous forme pulvérisée.

combustion - combustion

Réaction d'oxydation exothermique mise en œuvre dans certains moteurs thermiques pour réaliser un cycle thermodynamique, plus particulièrement pour apporter au fluide moteur la chaleur qui sera transformée en travail. Elle est intermittente ou continue selon que le moteur est une machine volumétrique à combustion interne ou non.

combustion diesel - diesel combustion

Processus de combustion caractéristique du fonctionnement des moteurs diesels, se déroulant en présence d'un excès d'air (25 à $30 \%$ ) et selon quatre phases distinguables qui sont : la phase du délai d'allumage ; la phase de combustion détonante; la phase de combustion contrôlée; la phase de combustion tardive.

combustion isochore - isometric combustion

Combustion se déroulant à volume constant. Il s'agit notamment du type de combustion mis en œuvre dans les moteurs à explosion, ou moteurs à allumage commandé, qui fonctionnent suivant le cycle de Beau de Rochas. La course décrite par le piston à l'intérieur du cylindre pendant le temps que dure la combustion explosive est très petite. Sur le diagramme dynamique ou diagramme $\mathrm{p}-\mathrm{v}$, la transformation correspondante est représentée par un segment de droite parallèle à l'axe des pressions.

compression - compression

Transformation thermodynamique que l'on fait subir à un gaz et qui a pour effet d'en augmenter la pression par apport d'énergie mécanique, donc par diminution du volume spécifique. Lorsque la transformation est adiabatique (le gaz étant isolé thermiquement du milieu extérieur), elle s'accompagne d'un accroissement de la température. Dans les moteurs thermiques, la compression est une transformation du cycle contribuant à rétablir ou à entretenir les conditions de la détente.

cycle (à combustion) à volume constant - constant volume (combustion) cycle Expression utilisée parfois pour désigner de façon descriptive le cycle de Beau de Rochas.

cycle à compression préalable

Tout cycle thermodynamique utilisé pour produire du travail, dans lequel le rétablissement des conditions de la détente met en jeu une compression.

cycle de Beau de Rochas - Otto's cycle

Cycle thermodynamique selon lequel fonctionnent les moteurs à explosion. Dans ce cycle, la détente se fait selon un processus adiabatique, et le retour à l'état initial met en jeu successivement : un refroidissement à volume constant qui commence avec l'ouverture de la capacité de détente (ouverture de la soupape d'échappement) et permet d'amener le fluide moteur à la température de la source froide (milieu ambiant); une compression adiabatique, qui fait retrouver au fluide moteur son volume spécifique initial en même temps qu'il restitue la chaleur non transformable en travail à la source froide; et un 
échauffement à volume constant (combustion isochore) dont l'effet est de ramener le fluide moteur à la pression et à la température initiales.

cycle de Carnot - Carnot's cycle

Cycle thermodynamique idéal selon lequel la production de travail se fait à l'occasion de deux détentes réversibles (une détente isotherme pendant laquelle se fait l'apport de chaleur à partir de la source chaude à température constante, suivie d'une détente adiabatique amenant le fluide moteur à la température de la source froide) et selon lequel le retour aux conditions initiales met en jeu deux compressions également réversibles (une compression isotherme pendant laquelle le fluide moteur cède à la source froide la chaleur reçue non transformable en travail, suivie d'une compression adiabatique ramenant le fluide moteur à la température de la source chaude en même temps qu'à son volume spécifique initial).

cycle de Joule - Brayton's cycle

Cycle thermodynamique selon lequel fonctionne la turbine à gaz. L'apport de chaleur coïncide avec la première partie de la détente qui s'effectue à pression constante et se trouve ainsi freinée. Une détente adiabatique amène ensuite le fluide moteur à la pression atmosphérique. Le retour aux conditions initiales met en jeu d'abord un refroidissement à la pression atmosphérique grâce auquel le fluide moteur se rapproche du volume spécifique initial et atteint une température inférieure à la température de début de détente; ce refroidissement est suivi d'une compression adiabatique.

\section{cycle de Lenoir}

Cycle thermodynamique comportant trois transformations seulement : une détente polytropique au cours de laquelle du travail est produit et qui amène le fluide moteur à la pression atmosphérique ; un refroidissement à pression constante le faisant revenir au volume de départ ; et un échauffement à volume constant qui rétablit les conditions initiales de la détente (augmentation de la pression et de la température à volume constant par apport de chaleur grâce à une explosion par exemple). Le fait que le fluide moteur ne soit pas comprimé avant de recevoir de la chaleur limite le rendement maximal de ce cycle, qui n’a pas survécu à son auteur et dont l'intérêt n'est qu'historique.

cycle de Stirling - Stirling's cycle

Cycle thermodynamique idéal selon lequel on s'efforce de faire fonctionner des moteurs à combustion externe d'un type très particulier. Il comporte deux transformations isothermes (détente et compression) réunies par deux transformations isochores (refroidissement et échauffement à volume constant). On peut considérer ce cycle comme un cycle de Carnot où les adiabatiques réunissant les isothermes seraient remplacées par des transformations à volume constant.

cylindre - cylinder

Type particulier d'organe de détente, constitué par une capacité fermée de forme cylindrique à l'intérieur de laquelle se meut un piston jouant le rôle d'organe moteur.

\section{cycle thermodynamique - thermodynamic cycle}

Suite de transformations thermodynamiques subies par un fluide entre deux passages successifs par le même état. Au cours de ces transformations, le fluide échange de la chaleur et du travail avec le milieu extérieur. Au plan énergétique global, le bilan est nécessairement nul. Le fluide pourra cependant avoir, par exemple, absorbé plus de cha- 
leur qu'il n'en a restitué au milieu extérieur. Il aura alors dû fournir à ce dernier plus de travail qu'il n'en aura reçu de lui. Dans ces conditions, du point de vue du milieu extérieur, de la chaleur aura disparu pour réapparaître sous forme de travail.

\section{déflagration - deflagration}

Régime caractérisant une explosion, c'est-à-dire une combustion évoluant hors de tout équilibre thermodynamique, selon lequel la vitesse de propagation de la flamme au sein du mélange combustible est subsonique. Ce régime est celui de la combustion mise en œuvre dans les moteurs à allumage commandé, encore dits moteurs à explosion. À partir d'un point du mélange où se produit l'allumage initial, une onde de combustion, de forme sphérique et centrée sur ce point, s'éloigne progressivement et enflamme par transmission de chaleur les couches de gaz successives, sans qu'il y ait création d'une onde de choc comme dans le cas de la détonation.

détente - expansion

Augmentation du volume occupé par un fluide, s'accompagnant en général d'une diminution de sa pression. La détente est isotherme si elle s'effectue à température constante ; adiabatique si elle se déroule sans échange de chaleur avec l'extérieur; et polytropique de façon générale. Dans les moteurs thermiques, la détente d'un fluide moteur, auquel on a préalablement apporté de la chaleur, est utilisée le plus souvent pour actionner un organe mécanique moteur, roue de turbine ou piston, disposé à l'intérieur d'une enceinte ouverte ou fermée.

\section{détonation - detonation}

Régime caractérisant une explosion, c'est-à-dire une combustion évoluant hors de tout équilibre thermodynamique, selon lequel la vitesse de propagation de la flamme au sein du mélange combustible est supersonique. Au lieu de se propager par transmission de la chaleur, le phénomène de combustion progresse sous l'effet d'un phénomène mécanique revêtant la forme d'une onde de choc. Ce régime est celui de la phase principale de la combustion diesel, dite phase de combustion détonante.

\section{diagramme - diagram}

Graphique représentant l'évolution d'un système par les variations d'une grandeur caractéristique en fonction d'une ou de plusieurs autres grandeurs.

\section{diagramme pression-volume - pressure-volume diagram}

Diagramme utilisé pour représenter l'évolution d'un fluide considéré comme un système thermodynamique, sur lequel on porte le volume spécifique en abscisses et la pression en ordonnées. L'avantage de ce diagramme tient principalement au fait que les échanges de travaux entre le système et le milieu extérieur y sont figurés par des surfaces.

\section{Synonyme(s) : diagramme dynamique, diagramme de Clapeyron.}

\section{combustion isochore - isometric combustion}

Combustion se déroulant à volume constant. Dans le cycle de Beau de Rochas, l'apport de chaleur se fait grâce à une combustion de ce type, qui correspond à une transformation figurée sur le diagramme dynamique par un segment de droite parallèle à l'axe des pressions. 
distribution - distribution

1. Action d'alimenter en fluide moteur l'organe de détente d'un moteur thermique, et plus précisément d'un moteur thermique de type volumétrique où ce processus est discontinu et doit s'accomplir en synchronisme avec les mouvements de l'organe moteur (qui est alors un piston).

2. Mécanisme assurant la fonction de distribution.

Obs. : Ce terme est employé souvent dans les textes de façon absolue, c'est-à-dire sans que soit précisé ce qui fait l'objet de la distribution, et c'est là pour le profane une source d'ambiguité.

échanger (des travaux, de la chaleur) - exchange, to

Pour un système thermodynamique comme un fluide, recevoir du travail ou de la chaleur du milieu extérieur, ou lui en fournir.

énergie - energy

Propriété d'un système mesurant son aptitude à fournir du travail (au milieu extérieur). Dans un système isolé, il ne peut y avoir création ou destruction d'énergie, mais la forme de l'énergie peut changer.

énergie cinétique - kinetic energy

Pour un fluide thermodynamique (gaz ou vapeur), somme des énergies associées aux mouvements des particules qui le composent. Elle est égale au travail qu'il faudrait dépenser pour les amener au repos et constitue une composante de l'énergie interne.

Obs. : On emploie quelquefois l'expression "énergie de vitesse".

énergie interne - internal energy

Pour un gaz (utilisé comme fluide thermodynamique), somme de l'énergie cinétique de ses molécules et de son énergie de configuration, c'est-à-dire de la somme des travaux des forces de liaison intermoléculaire.

énergie (potentielle) de pression - pressure energy

Travail que produiraient les forces qu'exercent les molécules d'un gaz les unes sur les autres si ce dernier était amené dans un état correspondant à celui d'un gaz parfait. Dans un moteur thermique, on recueille cette forme d'énergie à l'occasion de la détente du gaz que constitue le fluide moteur, directement ou après l'avoir transformée en énergie cinétique.

\section{évolution}

Synonyme de transformation.

explosion - explosion

Combustion évoluant hors de tout équilibre thermodynamique. C'est une réaction de combustion qui s'emballe. Deux régimes d'explosion types sont à distinguer : la déflagration et la détonation.

fluide moteur - working fluid

Dans un moteur thermique, fluide recevant de l'énergie sous forme de chaleur d'une source chaude et la restituant en partie sous forme de travail à l'organe moteur (piston ou roue de turbine) d'un appareil de détente et en partie sous forme d'énergie thermique résiduelle à une source froide. Le fluide moteur décrit un cycle thermodynamique, c'est- 
à-dire qu'il subit - à partir d'un état initial défini par les valeurs de deux grandeurs physiques (la température et la pression, par exemple) - une suite de transformations qui le ramènent à l'état de départ.

front de flamme - flame front

Dans un milieu gazeux où se propage la combustion à partir d'un point d'inflammation, surface formée par les points atteints par la flamme à un instant donné.

grandeur physique - physical quantity

Propriété physique à laquelle on peut faire correspondre une valeur numérique.

hyperbole - hyperbola

Courbe déterminée par l'intersection d'un cône avec un plan quelconque, si ce dernier coupe les deux nappes du cône.

hyperbole équilatère - rectangular hyperbola, equilateral hyperbola Hyperbole dont les asymptotes sont perpendiculaires l'une à l'autre.

\section{inflammation}

Synonyme d'allumage.

intégrale (d'une fonction) - integral

Résultat de l'intégration (inverse de la dérivation) d'une fonction $\mathrm{f}(\mathrm{x})$ sur un intervalle défini, que représente l'aire limitée par la courbe traduisant $\mathrm{f}(\mathrm{x})$, l'axe des abscisses et les deux droites parallèles à l'axe des ordonnées qui passent par les limites de l'intervalle.

isobare - isobaric

Se dit d'une transformation thermodynamique qui se déroule à pression constante. Sur le diagramme pression-volume, une telle transformation est représentée par un segment de droite parallèle à l'axe des volumes.

isochore - isometric

Se dit d'une transformation thermodynamique qui s'effectue à volume constant. Sur le diagramme pression-volume une telle transformation est représentée par un segment de droite parallèle à l'axe des pressions. Elle résulte d'un échange de chaleur du système (fluide) avec le milieu extérieur. Il peut s'agir d'un échauffement avec augmentation de la pression, ou d'un refroidissement avec baisse de la pression.

\section{isotherme - isothermal}

Se dit d'une transformation à température constante.

machine thermique

Machine conçue pour mettre en œuvre un cycle thermodynamique et utilisée pour convertir de la chaleur en travail ou l'inverse.

machine thermique motrice Synonyme de moteur thermique.

mécanisme de distribution Voir distribution. 
milieu extérieur - surroundings, external world

En thermodynamique, expression utilisée pour désigner tout ce qui ne fait pas partie du système thermodynamique à l'évolution duquel on s'intéresse.

moteur à allumage commandé - ignition-controlled engine

Moteur à combustion intermittente dans lequel l'amorçage périodique de la combustion destinée à réaliser un apport de chaleur se fait grâce à une étincelle électrique produite par un appareil d'allumage dont le fonctionnement est synchronisé avec celui de l'organe moteur. S'oppose à moteur à allumage par compression.

Synonymes : moteur à allumage par étincelle, moteur à explosion.

moteur à allumage par étincelle

Synonyme de moteur à allumage commandé.

moteur à combustion

Terme employé autrefois comme synonyme de moteur diesel. Au début du diesel, il évoquait adéquatement la différence avec le moteur à explosion quant au régime de propagation de la flamme, du fait que tous les moteurs diesels étaient des machines lentes où le combustible brûlait au fur et à mesure de son introduction, sans explosion et en général sans augmentation brusque de pression.

moteur à combustion externe - external combustion engine

Moteur thermique dans lequel l'apport de chaleur au fluide moteur se fait à l'extérieur de l'organe où la chaleur est convertie en travail, c'est-à-dire à l'extérieur de l'organe de détente.

moteur à combustion intermittente - intermittent combustion engine

Moteur à combustion interne dans lequel la détente se fait à l'intérieur d'une capacité fermée, qui sert en outre de foyer de combustion.

moteur à combustion interne - internal combustion engine, i.c. engine

Moteur où l'apport de chaleur au fluide moteur se fait grâce à une combustion à laquelle ce dernier participe en tant que comburant.

moteur à essence - gasoline engine, petrol engine

Moteur à allumage commandé utilisant l'essence comme carburant.

moteur à gaz - gas engine

Moteur à allumage commandé dans lequel un gaz combustible est brûlé à la place d'essence.

moteur à piston(s) - piston engine

Moteur à combustion intermittente où l'organe moteur est un piston se déplaçant à l'intérieur d'un cylindre.

moteur cinétique - non-displacement type engine

Moteur thermique où la détente du fluide moteur se fait à l'intérieur d'un organe ouvert selon un processus continu. La turbine à gaz et la turbine à vapeur sont des exemples de moteurs cinétiques. 


\section{moteur de Stirling - Stirling engine}

Moteur à combustion externe de type à récupération, qui réalise le cycle de Stirling. Cette machine est originale dans la mesure où le fluide moteur, qui est réutilisé indéfiniment, oscille entre une zone maintenue à haute température où il se détend de façon isotherme en recevant de la chaleur de la source chaude et une zone à basse température où il est comprimé de façon isotherme en même temps qu'il restitue à la source froide la chaleur non transformable en travail. Durant le transvasement de la zone chaude à la zone froide, le fluide moteur subit un refroidissement isochore (à volume constant). Il cède des calories à un milieu récupérateur qui les lui restituera lors de la remontée de l'échelle des températures à l'occasion du transvasement de retour.

\section{moteur diesel - diesel engine}

Moteur à combustion intermittente (c'est-à-dire à combustion interne et de type à capacité de détente fermée), se distinguant par le fait que son cycle met en jeu une très forte compression préalable. Un tel choix oblige à introduire le combustible seulement en fin de compression, mais dans un air suffisamment chaud pour qu'il s'enflamme de façon spontanée et $n$ 'impose pas le recours à une source d'énergie auxiliaire pour l'allumage.

Synonymes : moteur à combustion (vieilli), moteur à allumage par compression, moteur à injection de gazole, moteur à huile lourde.

moteur volumétrique - positive displacement engine, displacement type engine Moteur thermique utilisant l'énergie potentielle d'un gaz (le fluide moteur) que l'on fait se détendre, pour en tirer du travail, à l'intérieur d'une enceinte fermée dont le volume est variable. ble.

Synonyme : moteur à capulisme, machine volumétrique, moteur à capacité varia-

organe de détente - expander, expanding unit

Dans une installation ou une machine thermique motrice, organe réalisant une conversion thermodynamique où, par détente d'un fluide moteur, la chaleur cédée au préalable à ce dernier par une source chaude se trouve en partie transformée en travail.

\section{organe moteur}

Partie de l'organe de détente d'une machine thermique motrice qui reçoit la poussée résultant de la détente du fluide moteur. Il s'agit d'un piston ou d'une roue de turbine.

piston - piston

Dans un moteur à combustion interne à pistons, organe mécanique moteur de forme cylindrique et creux le plus souvent, sur la tête duquel s'exerce la poussée engendrée par la détente des gaz brûlés consécutive à la combustion du mélange carburé.

polytropique - polytropic

Se dit de toute transformation subie par un gaz, qui ne se distingue pas par la constance d'un paramètre d'état (pression, température, entropie, volume, etc.).

pompe à chaleur - heat pump

Machine thermique où un fluide subit un cycle thermique au cours duquel du travail est utilisé pour faire passer de la chaleur d'une source froide à une source chaude, c'est-àdire pour réchauffer un milieu chaud en extrayant des calories d'un milieu froid. 
rapport volumétrique de compression - compression ratio

Pour un moteur à combustion interne de type volumétrique, rapport entre le volume de la charge en début de compression et le volume qu'elle occupe à la fin de la course de compression.

Synonymes : rapport de compression, taux de compression.

rendement - efficiency

Nombre mesurant l'efficacité d'une transformation ou d'une machine transformatrice d'énergie. De façon générale, c'est le quotient de l'énergie utile recueillie à l'énergie fournie pour l'obtenir dont on ne dispose pas gratuitement. Dans le cas d'un moteur thermique, il s'agit du rapport de la chaleur apportée par le combustible au travail utile recueilli sur l'organe moteur primaire ou sur l'arbre moteur, selon le type de rendement considéré.

rendement thermodynamique - maximum theoretical efficiency, availability factor, Carnot fraction

Pour un moteur thermique, quotient du travail qui serait produit par la machine si toutes les transformations s'y déroulant étaient réversibles, à l'énergie fournie au fluide moteur sous forme de chaleur.

Synonymes : rendement de Carnot, rendement thermodynamique théorique.

réversible - reversible

Qualifie une transformation idéale qui, à partir d'un état initial, amènerait un système à un état final en le faisant passer par une succession continue d'états correspondant chacun à un équilibre du système.

source chaude - hot body, heat source

Milieu auquel le fluide moteur mis en œuvre dans une machine thermique motrice emprunte les calories qui seront transformées en travail au cours d'une détente.

source froide - cold body, cold reservoir, heat sink, heat absorber

Milieu auquel un moteur, après avoir transformé en travail une partie de la chaleur reçue d'une source chaude, restitue la chaleur résiduelle qui ne peut être convertie en énergie mécanique d'après le principe de Carnot.

source thermique - thermal source

Corps ou milieu extérieur à un système qui, possédant une capacité thermique pratiquement infinie, peut échanger avec ce dernier, sans modification de sa température, une quantité de chaleur quelconque.

Synonyme : thermostat.

taux de compression

Synonyme de rapport volumétrique de compression.

travail - work

Grandeur physique mesurant l'effet utile d'une force, qui est égale au produit de la distance dont s'est déplacé le point d'application de la force par la projection de la force sur la direction de déplacement. 
volume spécifique - specific volume

Volume occupé par l'unité de masse d'une substance, d'un gaz notamment.

JACQUES LETHUILLIER Université de Montréal, Montréal, Québec, Canada 